\title{
Efecto de la inoculación de micorrizas arbustivas en la evaluación agronómica de cuatro tipos de pastos en el cantón Pedernales, provincia de Manabí, Ecuador.
}

\section{Effect of shrub mycorrhizal inoculation in the agronomic evaluation of four varieties of grasses in the canton Pedernales province of Manabí. Ecuador.}

(iD) 'Elber Vinicio Solís Canchingre (iD 'Henrry Othón Intriago Mendoza iD ${ }^{2}$ Alfredo Noa Monzón
(iD) 'Tyrone Antonio Zambrano Barcia

Universidad Laica "Eloy Alfaro" de Manabí, extensión Pedernales, Ecuador Jardín Botánico, Universidad Central "Marta Abreu" de Las Villas, Cuba,

\section{Resumen}

En el Ecuador la actividad ganadera tiene un rol importante, es conocido que la alimentación del ganado básicamente es en torno a las gramíneas, por ser el alimento natural y de bajo costo que crece en los potreros. Evaluar los datos agronómicos de los pastos con la inoculación de micorrizas arbusculares y contrastar con las gramíneas que no fueron inoculadas, es de importancia ya que se logró obtener información sobre si es conveniente el uso de estos hongos para mejorar los rendimientos agronómicos en los pastos tropicales. Los datos que se evaluaron son altura de planta, diámetro de tallo, largo de hoja, ancho de hoja, peso de planta (g), rendimiento kg/ha, las gramíneas sometidas a esta investigación son: Brizanta, Urochloa brizantha (Hochst. ex A. Rich.) R. D. Webster; Saboya, Panicum maximum Jacq.; Tanzania, Panicum maximum cv. Tanzania) y Pangola, Digitaria eriantha Steud. Todos los taxones con que se experimentó tienen una dependencia de micorrizas de aproximadamente el 80\%.

Palabras clave: Evaluación agronómica, pastos, micorrizas arbusculares

Recibido:

Mayo 2020

Aceptado:

Setiembre 2020

\section{Abstract}

In Ecuador, livestock activity has an important role it is known that livestock feeding is around grasses, as it is the natural and low-cost food that grows in paddocks. Evaluating agronomic data of grasses with inoculation of arbuscular mycorrhizae and contrast with the grasses that were not inoculated, it is important because it somehow obtains information about whether to use these fungi to improve biomass yields on pasture tropical, the data are evaluated plant height, stem diameter, leaf length, leaf wi$\mathrm{dth}$, plant weight (g), yield kg/ha, grasses subjected to this research are: Palisade grass, Urochloa brizantha (Hochst. ex A. Rich.) R. D. Webster; Guinea grass, Panicum maximum Jacq; Tanzania Guinea grass, Panicum maximum cv. Tanzania and Pangola grass, Digi- 
taria eriantha Steud. Are species with mycorrhizal dependence of approximately 80\%.

Keywords: Agronomic evaluation, grasses, arbuscular mycorrhizae

\section{INTRODUCCIÓN}

La ganadería es un sector clave en la economía de los países de América Latina Tropical, pues ocupa una amplia fracción de los recursos de la tierra con potencial productivo, En el Ecuador, la superficie con uso agropecuario oscila alrededor de 12'355.881 ha, de las cuales 3'357.167 ha, corresponden a pastos cultivados en 298.962 Unidades de Producción Agropecuaria (UPAs) y 1'129.701 ha, de pastos naturales, lo que significa que el $36 \%$ del suelo de uso agropecuario están ocupadas por pastos (INEC, 2013).

Entre los principales pastos cultivados en el Ecuador se destacan las braquiarias, el pasto Elefante, pasto Tanzania, Saboya, pasto Estrella, entre otras. Los beneficios que se obtienen de las mejoras de los pastos son a mediano y largo plazo, debido a los cambios sustanciales en las condiciones económicas (cambios de precios) ambientales (plagas, enfermedades, sequías), que determinan que la decisión de invertir en pastura involucre un alto nivel de riesgo.

Se conoce como micorrizas a la asociación mutualista establecida entre las raíces de las plantas con ciertos hongos del suelo, produciendo una simbiosis en las cuales las dos se favorecen mutuamente, en este caso, micorrizas arbusculares (Pérez, Rojas y Montes, 2011). Por otra parte, la explotación ganadera eficiente, bien sea de carne o leche, exige una mayor demanda de la calidad de alimentación, por lo que el valor nutritivo de las especies forrajeras, sus condiciones naturales, su manejo, aplicación correcta de fertilizantes y corte oportuno, deben ser investigados por regiones, ya que las condiciones ambientales son diferentes. (Acosta, 2005).

La provincia de Manabí tiene la mayor superficie del país con uso agropecuario de la tierra con 1'171.273 ha, así como las dedicadas a pastos, de ellas 719.883 ha, corresponden a pastos cultivados y 166.750 ha a pastos naturales lo que se corresponde con la mayor población bovina, 911.748 cabezas de ganado (INEC, 2013).

Entre las especies de gramíneas forrajeras existentes en esta región, se encuentran Brizanta (Urochloa brizantha); Saboya (Panicum maximum); Tanzania (Panicum maximum cv. Tanzania) y Pangola (Digitaria eriantha). Estos pastos sirven como alimento de los animales en todas las épocas del año, permitiendo un aporte nutricional que beneficia a la condición corporal, la producción y al estado de salud de los animales. Con la inoculación de micorrizas arbusculares en el cultivo de dichas especies, se logra un incremento del valor nutritivo para los animales al crecer más vigorosas, lo cual ha sido reportado en investigaciones precedentes como alternativa de mejora alimenticia, por lo que el objetivo del presente estudio fue evaluar los parámetros de crecimiento vegetativo de las gramíneas mencionadas bajo las condiciones ambientales de la costa manabita y con el empleo del inóculo con que se experimenta. Los mayores problemas de los suelos de Manabí especialmente los que tienen cercanía a los sectores de Pedernales, presentan niveles de medios a bajos de fósforo, siendo estos valores inferiores a 14 ppm en el sitio experimental, lo que conlleva una limitación en el crecimiento radicular y transferencia de energía de la planta, desde luego ocasionando una baja fotosíntesis y productividad.

\section{MATERIALES Y MÉTODOS}

Las especies de gramíneas forrajeras que se estudiaron fueron: Urochloa brizantha; Panicum maximum; Panicum maximum cv. Tanzania y Digitaria eriantha. Se profundizó en los caracteres botánicos, ecológicos y de distribución de las especies de gramíneas objeto de estudio y en las características de la rizosfera, que comprende la región del suelo ocupada por las raíces de 
las plantas, donde crece una comunidad microbiológica diversa y dinámica, cuya actividad se vincula con distintos procesos relacionados con el agua, nutrición mineral, intercambio de cationes y producción de exudados, entre muchos otros, que la hacen diferente del resto del suelo en sus propiedades físicas, químicas y biológicas (Reyes, 2011).

Para medir la altura de la planta, el largo y el ancho de las hojas se utilizó una cinta métrica; para el diámetro del tallo un calibrador; el peso de la planta se determinó con el empleo de una balanza gramera Marca Denver, Modelo APx200, Serie 2408-2038. Para el cálculo del rendimiento por hectárea, se cosechó dentro de cada parcela un metro cuadrado a los 45, 70 y 95 días, luego se pesó expresando el valor en $\mathrm{kg} / \mathrm{ha}$. Los dos tratamientos fueron inoculados uno, con 0,15 g y otro con 0,30 g, de micorrizas arbusculares, se dejó un trata- miento testigo sin inóculo. Los datos tomados in situ al azar de 10 plantas fueron: altura de planta, diámetro del tallo, largo de hoja y ancho de hoja, esto se realizó a los 30, 45, 70 y 95 días. El peso de la planta se midió a 45, 75, 95 días y se promedió; el primer corte se realizó a $10 \mathrm{~cm}$ de altura, los demás a $15 \mathrm{~cm}$. Para calcular el rendimiento en $\mathrm{kg} / \mathrm{ha}$ se cosechó una parcela de un metro cuadrado.

\section{Tratamiento y Diseño Experimental}

Se evaluó el efecto de cuatro tipos de gramíneas: Digitaria eriantha, Urochloa brizantha, Panicum maximum Panicum maximum $\mathrm{cV}$. Tanzania, con 12 tratamientos y 4 repeticiones; a los tratamientos se les inoculó $0,15 \mathrm{~g}$ y $0,30 \mathrm{~g}$ de micorrizas arbuscular, excepto al testigo. Las mediciones experimentales se evaluaron bajo un Diseño de Bloques Completamente al Azar (DBCA) con factorial AxB (ver Tabla 1)

\section{Tabla 1}

Esquema del análisis de varianza.

\begin{tabular}{ccc}
\hline F. VARIANZA & FóRMULA & GRADOS DE LIBERTAD \\
\hline Total & r.t-1 & 47 \\
Repeticiones & $\mathrm{r}-1$ & 3 \\
Factor A & $\mathrm{a}-1$ & 3 \\
Factor B & $\mathrm{b}-1$ & 3 \\
Interacción AxB & $(\mathrm{a}-1)(\mathrm{b}-1)$ & 6 \\
Error & $(\mathrm{r}-1)(\mathrm{t}-1)$ & 33 \\
\hline
\end{tabular}

\section{Localización y duración del experimento}

El presente estudio se realizó en el sitio Chiquimble del cantón Pedernales, Provincia de Manabí; ubicado geográficamente en $02^{\circ} 43^{\prime} 34^{\prime}$ de Latitud Norte y $02^{\circ} 43^{\prime} 35^{\prime}$ de Latitud Sur, a $89^{\circ} 59^{\prime} 5^{\prime}$ de Longitud Este y a $0^{\circ} 0^{\prime} 0^{\prime}$ de
Longitud Oeste con una altitud de 100 msnm.

En la tabla 2, se registran las condiciones meteorológicas y en la tabla 3 las condiciones edafológicas y del relieve de la zona donde se realizó la investigación. 


\section{Tabla 2}

\section{Condiciones meteorológicas}

\begin{tabular}{cc}
\hline Parámetro & Promedio \\
\hline Pluviosidad anual & $800 \mathrm{~mm}$ \\
Heliofanía anual & $2160 \mathrm{horas}$ \\
Temperatura promedio & $23,13^{\circ} \mathrm{C}$ \\
Evaporación anual & $229,01 \mathrm{~mm}$ \\
Temperatura suelo & $28,19^{\circ} \mathrm{C}$ \\
Velocidad del viento & $5,08 \mathrm{~km} / \mathrm{h}$ \\
Presión atmosférica & $1010,89 \mathrm{bar}$ \\
Humedad relativa & $82,40 \%$ \\
\hline
\end{tabular}

\section{Tabla 3}

Condiciones edafológicas y del relieve.

\begin{tabular}{cc}
\hline Topografia & Plana \\
\hline Textura suelo & Franco arcilloso \\
Drenaje & Natural \\
$\mathrm{pH}$ & 5.8 \\
\hline
\end{tabular}

\section{RESULTADOS Y DISCUSIÓN}

Descripción botánica de las especies estudiadas

Panicum maximum Jacq.

Familia Poaceae (Gramíneas)

Planta perenne, de la zona tropical y subtropical del centro del África, forma macollas, puede alcanzar hasta $3 \mathrm{~m}$, sus tallos son erectos $\mathrm{y}$ ascendentes con una vena central pronunciada y de 1 a 1,5 cm de diámetro, inflorescencia en forma de panoja abierta de 12 a $40 \mathrm{~cm}$ de longitud, raíces fibrosas largas y nudosas, ocasionalmente rizomatosas, lo que le confiere cierta tolerancia a la sequía. Requiere de suelos de media o alta fertilidad, bien drenados con $\mathrm{pH}$ de 5 a 8, no tolera suelos inundables, crece entre 0-1500 msnm y con precipitaciones entre 1000-3500 mm por año; crece muy bien en temperaturas altas, tolera media sombra y crece bien bajo árboles. Se establece por semillas con una tasa de siembra de 6-8 kg/ha, super- ficial y ligeramente tapada, el primer pastoreo se recomienda a los 90-120 días después de la siembra o bien antes de iniciar la floración, produce entre 10-30 t/ms/ha por año. Nombre común Guinea, Saboya. Biovirtual (2010)

\section{Digitaria eriantha Steud.}

Familia Poaceae (Gramíneas)

Planta perenne, procedente de Transvaal, sur de África; crece en áreas tropicales y subtropicales, produce estolones y unos tallos finos de hasta 2 m de longitud, cilíndricos, ramificados y de color rojizo, capaces de enraizar a partir de los nudos inferiores; sus hojas largas y estrechas pueden estar o no recubiertas de pelos; inflorescencia en forma de espiga, con las flores en espiguillas pareadas.

Se adapta a diversos ecosistemas, desde el nivel del mar hasta 1800 msnm, con precipitacio- 
nes entre 1000 a $3500 \mathrm{~mm}$ al año, temperaturas por encima de $19^{\circ} \mathrm{C}$, crece bien en regiones de baja fertilidad con sequias prolongadas, no soporta encharcamientos prolongados. La productividad de materia seca de esta especie es variable, dependiendo de las condiciones climáticas del año y la fertilidad del suelo, produce entre 12 y 29 t/ms/ha año. Se establece por semillas o en forma vegetativa, nombre común Pangola. Tropicalforanges (2011)

Urochloa brizantha (A. Rich.) R. D. Webster Familia Poaceae (Gramíneas)

Planta perenne originaria de África tropical, provista de tallos más o menos erectos de 1 a 1,5 m, produce rizomas cortos y hojas lanceoladas y pilosas, inflorescencia en racimo. Se adapta a climas de media fertilidad con rangos amplios de textura y pH 4 a 8; tolera alturas entre 0-1800 msnm y precipitaciones entre 1000$3500 \mathrm{~mm}$, sequias prolongadas, aunque no soporta encharcamientos mayores a 30 días, tiene buena persistencia a pastoreo y compite con las malezas. Presenta alta producción de forraje en rango amplio de ecosistemas y suelos, su producción anual varía de 8 a 30 t/ms/ha año; el pasto soporta altas cargas, la propagación es por semilla gámica o de forma vegetativa, se utiliza de $3-4 \mathrm{~kg} / \mathrm{ha}$, requiere una buena preparación del suelo y se siembra preferentemente al inicio de las lluvias. Nombre común Brizanta, Marandú. Ecuared (2012)

Panicum maximum cv. Tanzania

Familia Poaceae (Gramínea)
Planta tropical perenne originaria de Tanzania, África, bajo crecimiento libre puede alcanzar 2,5 m de altura y producir perfiles semi-decumbentes que enraízan; inflorescencia de coloración púrpura a medida que avanza la fase reproductiva de la planta. Se adapta desde el nivel del mar hasta los 1800 msnm, soporta niveles moderados de sequias, forma macollas, mejora su comportamiento cuando es sometida a penumbra o sombra rala de una especie arbórea adecuada, con sistemas de fertilización se han alcanzado niveles de producción de 40 a 50 t/ms/ha por año. Normalmente las praderas se establecen a través de semillas siendo necesario de 4 a $6 \mathrm{~kg} / \mathrm{ha}$. Nombre común: Tanzania. Mundo-Pecuario (2012)

\section{Micorrizas}

Familia Acaulosporaceae

Se conoce con el nombre de micorriza a la asociación mutualista establecida entre las raíces de la mayoría de las plantas y ciertos hongos del suelo, es una simbiosis prácticamente universal. Las raíces de las plantas terrestres proporcionan hábitat especializado donde los microorganismos obtienen todo o parte de sus requerimientos esenciales para su crecimiento, así; los hongos microrrizicos absorben nutrientes del suelo y liberan hacia las células hospedadoras. Las micorrizas utilizadas en la presente investigación se adquirieron en Bioamecsa (Bioquímicos de América S.A.) con el nombre comercial de Micorrizas Fungifert.

\section{Tabla 4}

Esquema del trabajo experimental.

\begin{tabular}{cccc}
\hline \multicolumn{1}{c}{ Pangola } & \multicolumn{1}{c}{ Brizanta } & Tanzania & Saboya \\
\hline A1xB1 sin micorrizas & A2xB1 sin micorrizas & A3xB1 sin micorrizas & A4xB1 sin micorrizas \\
A1xB2 0,15 g de micorrizas & A2xB2 0,15 g micorrizas & A3xB2 0,15 g micorrizas & A4xB2 0,15 g micorrizas \\
A1xB3 0,30 g de micorrizas & A2xB3 0,30 g micorrizas & A3xB3 0,30 g micorrizas & A4xB3 0,30 g micorrizas \\
\hline
\end{tabular}




\section{Análisis de las interacciones en la varia- ble altura de planta}

En la Figura 1 se muestra el efecto de las interacciones entre altura de planta y la aplicación de inóculo, siendo significativos los resultados. La mayor altura a los 30 días se alcanzó en la interacción Pangola con micorrizas 0,30 g/planta (A1xB3) con $8,82 \mathrm{~cm}$. A los 45 días también correspondió a esta planta, pero con micorrizas $0,15 \mathrm{~g} /$ planta (A1XB2) con 27,86 cm. A los 70 días el pasto Saboya resultó ser el de mayor altura sin aplicación de micorrizas (A4xB1) con $112,51 \mathrm{~cm}$, y a los 95 días, el pasto Tanzania sin aplicación de micorrizas (A3XB1) con 179,11 cm.

Por otra parte, la menor altura fue para el pasto Brizanta en los tratamientos sin apli- cación de micorrizas (A1xB1), con la aplicación de 0,30 g (A2xB3) y con la de 0,15 g (A2xB2) con alturas de $5,30 \mathrm{~cm} ; 12,50 \mathrm{~cm}$; $51,18 \mathrm{~cm}$ y $73,83 \mathrm{~cm}$, a los $30,45,70$ y 95 días respectivamente. Los resultados obtenidos en esta investigación son parecidos a los reportados por García (2017), quien concluye que la inoculación de Hongos Micorrícicos Arbusculares en plantas de banano, aportó beneficios significativos ayudando a mejorar el nivel nutricional de la planta, lo cual se ve reflejado en un mayor crecimiento de las plantas, aumentando la altura, sobresaliendo en todos los casos el tratamiento (15 g/planta), debido a la capacidad que presentan en la asimilación de nutrientes.

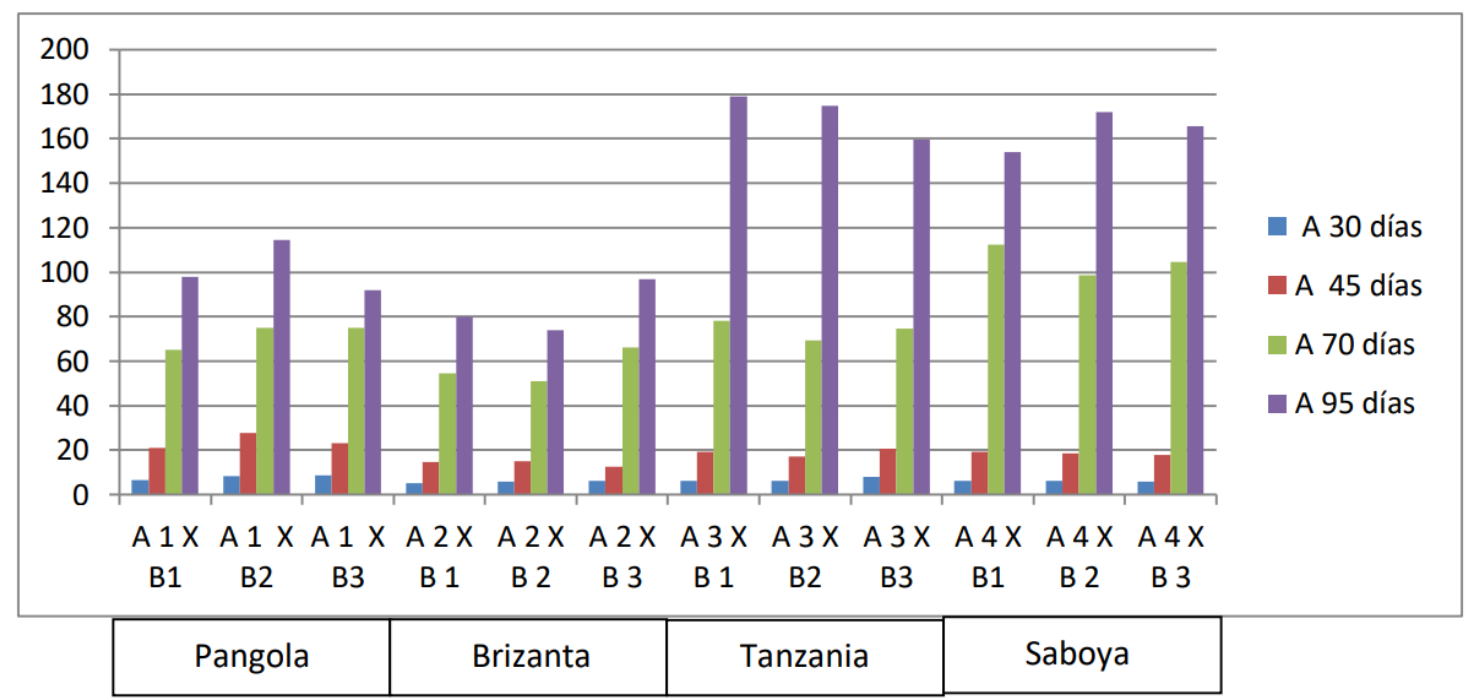

Figura 1. Efectos de las interacciones en la variable altura de planta (en cm) a los 30, 45, 70 y 95 días.

\section{Análisis de las interacciones en la varia- ble diámetro de tallo.}

En la Figura 2 se muestra el efecto de las interacciones entre diámetro de tallo de la planta y la aplicación de inóculo, no encontrándose alta significancia estadística, obteniéndose el mayor diámetro a los 30 días en el tratamiento del pasto Saboya con micorrizas 0,15 g/planta (A4xB2) con 3,47 mm, sobresaliendo a los 45, 70 y 95 días el pasto Saboya sin aplicación de micorrizas (A4xB1) con 9,50 mm; 10,16 mm; y 8,83 mm, respectiva- mente. Los de menor diámetro fueron el pasto Pangola sin aplicación de micorrizas (A1xB1) con 2,36 mm y 3,46 mm a los 30 y 45 días en su orden y el mismo pasto con micorrizas al 0,15 g/planta (A1xB2) con 3,37 mm y 3,42 mm a los 70 y 95 días respectivamente, lo que significa que se relaciona en pruebas realizadas en el cultivo del palmito. Según Paillacho (2010), las variables de crecimiento evaluadas: diámetro del tallo e índice de vigor, los mejores resultados se obtuvieron con la dosis de $20 \mathrm{~g}$ de inóculo de micorriza nativa 


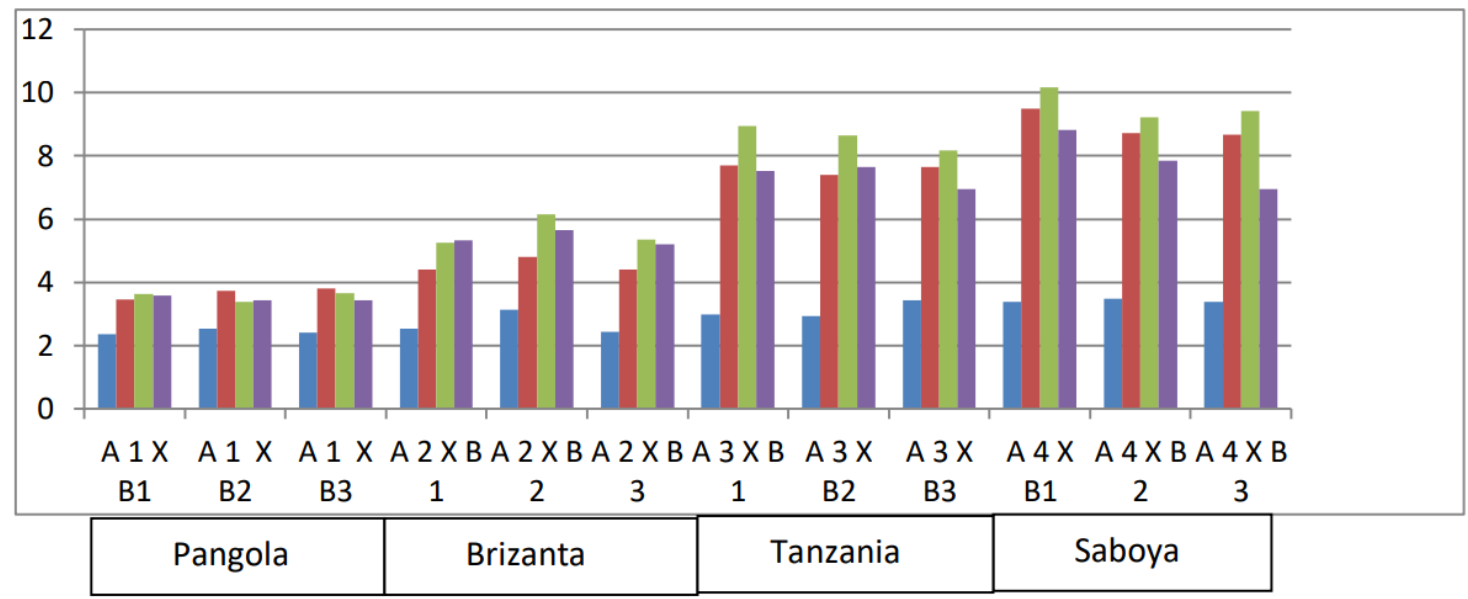

Figura 2. Efectos de las interacciones en la variable diámetro de tallo (en $\mathrm{mm}$ ) a los 30, 45, 70 y 95 días.

\section{Análisis de las interacciones en la varia- ble largo de hoja.}

En la Figura 3 se muestra el efecto de las interacciones entre largo de hoja y la aplicación de inóculo. A los 30 y 70 días se presentan diferencias estadísticas; a los 45 días no hubo diferencias estadísticas, mientras que a los 95 días se observó diferencia estadística, obteniendo el mayor valor del largo de las hojas en el pasto Tanzania con micorrizas $0,30 \mathrm{~g} /$ planta (A3xB3) con 19,76 cm; 45,19 cm; 90,93 cm y $76,01 \mathrm{~cm}$, a los 30, 45, 70 y 95 días respectivamente. Los menores valores se presentan en el pasto Saboya sin aplicación de micorrizas (A4xB1) con 13,69 cm a los 30 días, el pasto Pangola con micorrizas 0,30 g/planta
(A1xB3) con $27,34 \mathrm{~cm}$ y $36,75 \mathrm{~cm}$, a los 45 y 95 días, y el pasto Pangola sin aplicación de micorrizas (A1xB1) con 36,20 cm, a los 70 días, en su orden respectivo. Lo que se relaciona a lo manifestado con el estudio realizados por Maldonado y Reyes (2018). En plantas de tomate, las cepas de micorriza Acaulosphora sp. y Entrephosphora sp. tuvieron mayor eficiencia en el desarrollo de las variables de altura en semillero, días a floración, peso seco de raíz y tallo, donde se encontraron diferencias significativas. En plantas de pasto Marandú las cepas Glomus sp. y Acaulosphora sp. tuvieron mayor efecto en las variables de altura, macollamiento, peso seco de la planta y las características de las raíces.

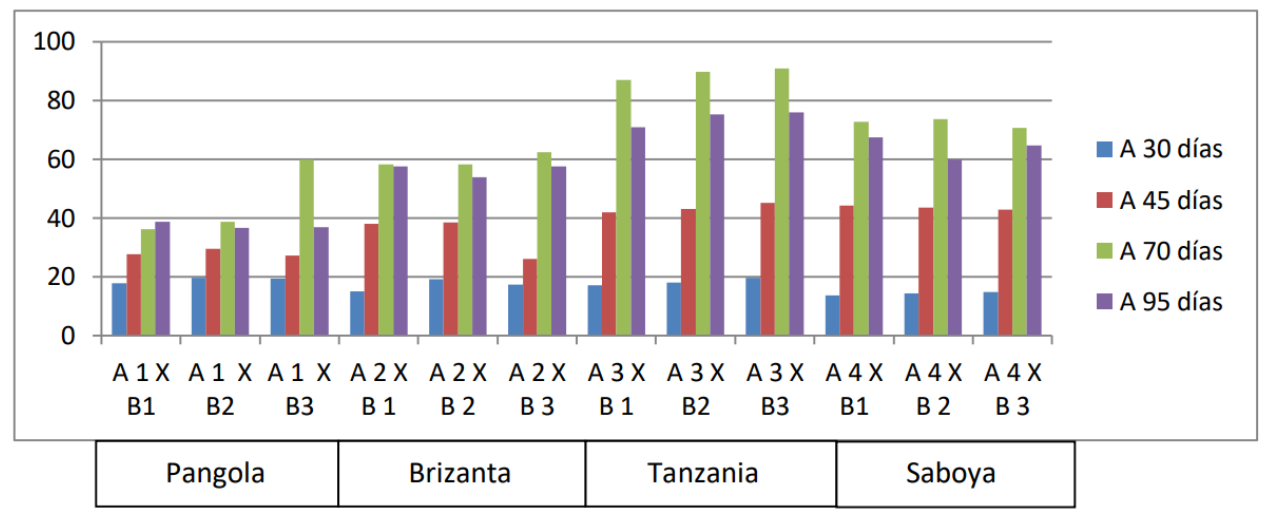

Figura 3. Efectos de las interacciones en la variable largo de hoja a los 30, 45, 70 y 95 días, en centímetros. 


\section{Análisis de las interacciones en el varia- ble ancho de hoja.}

En la Figura 4 se muestra el efecto de las interacciones entre ancho de hoja y la aplicación de inóculo. A los 30, 45, 70 y 95 días no se encontró diferencia significativa, presentando el valor mayor a los 30 días el pasto Brizanta con micorrizas 0,15 g/planta (A2xB2) con 1,24 cm, a los 45 y 70 días el pasto Saboya sin micorrizas (A4xB1) con 2,04 cm y 3,27 cm, respectivamente, y a los 95 días el pasto Tanzania con micorrizas 0,15g/planta (A3xB2) con 3,00 cm. Alcanzando los menores valores el pasto Tanzania sin micorrizas (A3xB1) con 1,02 cm a los 30 días, el pasto Pangola sin micorrizas (A1×B1) y Brizanta con micorrizas 0,30 g/planta (A2xB3) ambos con 1,67 cm a los 45 días, el pasto Pangola con micorrizas 0,30 g/planta (A1xB3) con 2,02 cm a los 70 días, el pasto Pangola sin micorrizas (A1xB1) y el mismo pasto con micorrizas 0,30 g/planta (A1xB3) ambos con 2,11 cm a los 90 días, cada caso en su orden. Lo cual concuerda en pruebas realizadas en Santo Domingo de lo Tsáchilas donde se estudió el efecto de la sustitución parcial de fertilizante químico por Zeolitas y micorrizas en la producción de Brócoli, investigación realizada por Reinoso (2014). Al analizar los resultados en las variables de prendimiento, crecimiento, longitud del tallo, diámetro del tallo, número de hojas, longitud de hoja y ancho de la hoja, se determinó que los que sobresalen son los tratamientos que fueron inoculados con micorrizas, esto confirma que éstas contribuyen a mejorar el crecimiento vegetativo, garantizando un mejor vigor y sobrevivencia de las plantas en el campo.

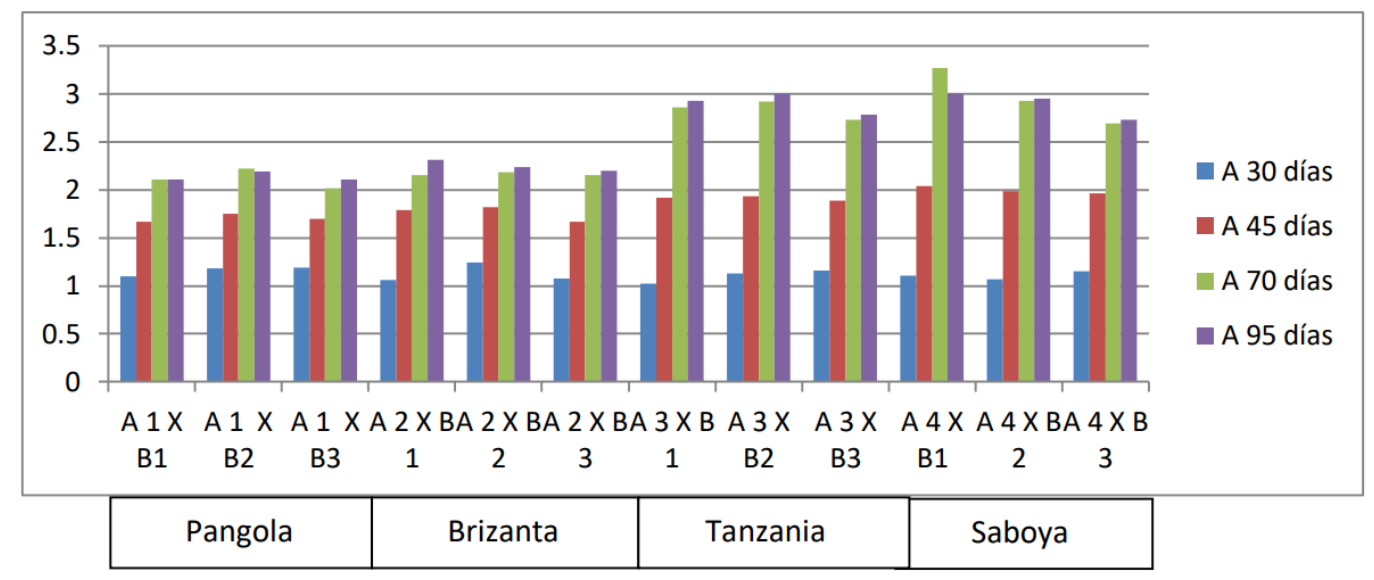

Figura 4. Efectos de las interacciones en la variable ancho de hoja a los 30, 45, 70 y 95 días, en centímetros.

\section{Análisis de las interacciones en la varia- ble peso de la planta.}

En la Figura 5, se muestra el efecto de la interacción entre peso de planta y aplicación de inóculo. A los 45 días, se encontró diferencias significativas, mientras que a los 70 y 95 días presentó alta significancia. Alcanzando el mayor peso el pasto Saboya sin micorrizas (A4xB1) con 10,8 g, $44 \mathrm{~g}, 35 \mathrm{~g}$ y $66,58 \mathrm{~g}$ a los 45,70 y 95 respectivamente, obteniendo los menores valores el pas- to Brizanta sin micorrizas (A2xB1) con 4,9 $\mathrm{g}$ a los 45 días y el pasto Pangola con micorrizas $0,30 \mathrm{~g} /$ planta (A1XB3) con 8,55 y 14,58 g/planta a los 70 y 95 días respectivamente. Estos resultados son comparables a los reportados por Prieto, Belezaca, Mora, Vallejo, Gutiérrez y Pinargote (2011), quienes corroboraron que el análisis varianza no mostró diferencias estadísticas significativas, tanto para el peso húmedo como para el peso seco del sistema foliar a los 78 días des- 
pués de las inoculaciones. Sin embargo, a los 103 días hubo diferencias estadísticas significativas entre los tratamientos, tanto para el peso húmedo ( $F=3.972, p=0.016)$ como para el peso seco $(F=3.306, p=0.031)$. Los tratamientos cuyas plantas fueron inoculadas con hongos de MA presentaron valores de peso estadísticamente superiores al tratamiento control.

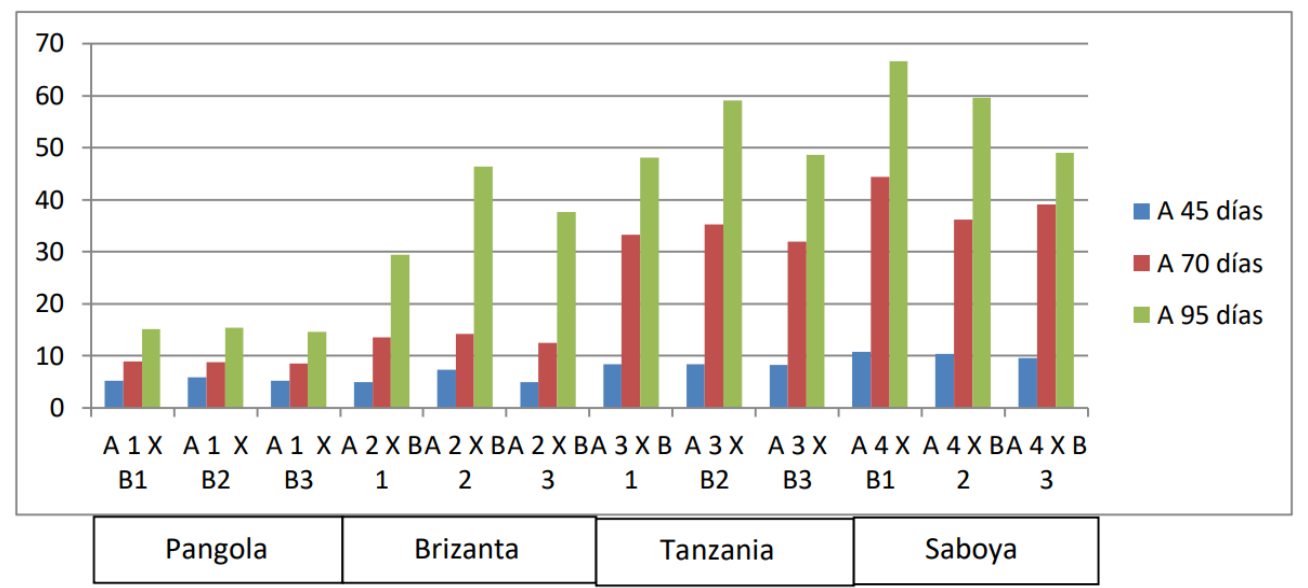

Figura 5. Efectos de las interacciones en la variable peso de planta 45, 70 y 95 días, en gramos.

\section{Análisis de las interacciones en la varia- ble rendimiento $\mathbf{k g} / \mathrm{ha}$.}

En la Figura 6, se observa el efecto de la interacción entre rendimiento kg/ha y la aplicación de inóculo. A los 45, 70 y 95 días se encontró alta significancia, al realizar la prueba de Tukey a los 45 y 95 días presentó cinco rangos de significancia, mientras que a los 70 días cuatro rangos de significancia, presentando el mayor valor el pasto Saboya sin micorrizas (A4xB1) con 7167 kg/ha, 29567 kg/ha y 44383 kg/ha a los 45, 70 y 95 días, en su orden. Por el contrario, los menores valores los presentó el pasto
Brizanta sin micorrizas (A2xB1) con $3267 \mathrm{~kg} / \mathrm{ha}$ a los 45 días, y el pasto Pangola con micorrizas 0,30 g/planta (A1xB3) con 5700 kg/ha y 9717 $\mathrm{kg} / \mathrm{ha}$ a los 70 y 95 días, cada caso en su orden, lo que concuerda con el estudio realizado por Noda (2009), quien manifiesta que la simbiosis micorrícica es un fenómeno ampliamente fundamentado y reconocido por la comunidad científica internacional, y no hay duda de los incrementos en la absorción de los nutrientes y el agua en las plantas micorrizadas; así como, de un mayor crecimiento y rendimiento de los cultivos.

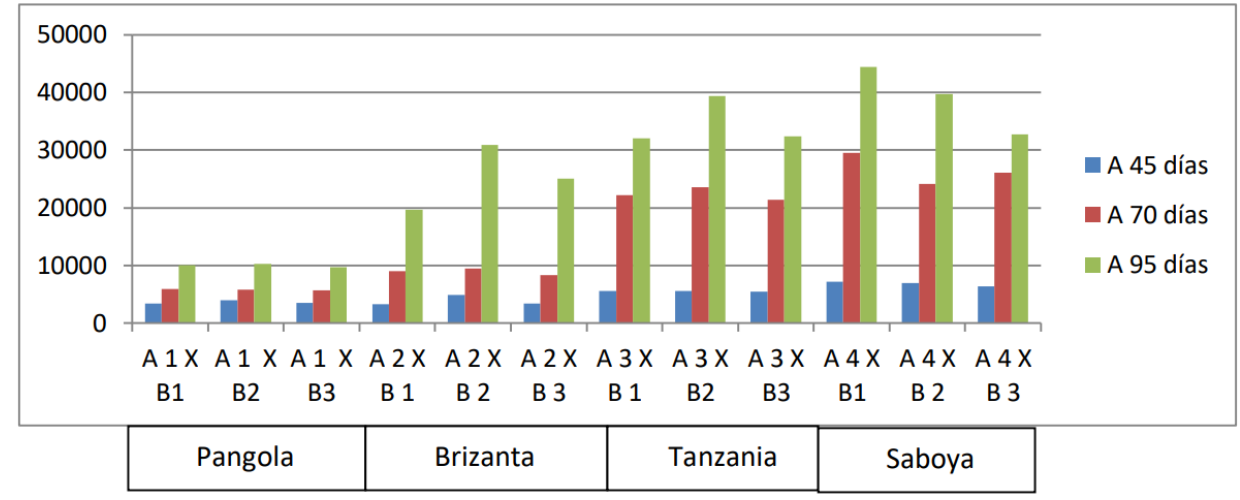

Figura 6. Efectos de las interacciones en la variable rendimiento $\mathrm{kg} / \mathrm{ha}$ a los 45,70 y 95 días. 


\section{CONCLUSIONES}

- Los pastos Pangola y Brizanta registraron las mejores alturas de plantas cuando se aplicó micorriza en dosis de 0,15 g/ planta y 0,30 g/ planta.

- De acuerdo a los datos obtenidos en altura de planta los pastos Saboya y Tanzania registran los mejores crecimientos cuando no se utilizaron las micorrizas.

- En diámetro del tallo se pudo evidenciar que el pasto Tanzania mostró el mejor índice de vigor de planta a los 70 días y 95 días utilizando 0,15 g/ planta.

- En la variable vigor de hoja se evidencia que Tanzania, Pangola y Brizanta con respuestas positivas a la aplicación de micorrizas de 0,15 / planta.

- El pasto saboya con respecto a la variable vigor de hoja no presenta respuesta positiva a la aplicación de micorrizas.

- El pasto Saboya sin aplicación de micorrizas presentaron valores superiores de productividad con promedios de $7167 \mathrm{~kg} / \mathrm{ha}, 29567$ kg/ha y $44383 \mathrm{~kg} / \mathrm{ha}$ a los 45, 70 y 95 días.

- El pasto Pangola con micorrizas 0,30 g/ planta tuvo rendimientos de $5700 \mathrm{~kg} / \mathrm{ha}$ y $9717 \mathrm{~kg} / \mathrm{ha}$ a los 70 y 95 días, como los de mejor incremento.

- Las variables respuestas con respecto a las micorrizas y su efecto en los primeros 30 días en las distintas variables biométricas donde se observa mejores incrementos, parecen estar relacionados con la presencia de hormonas en este caso auxinas.

\section{REFERENCIAS BIBLIOGRAFÍCAS}

Acosta, R. (2005). Los fertilizantes en un sistema de pastoreo rotativo, 4ta. Edición. Editorial FERTICA. México D.F. p15

Agrociencia. (2011). Estudio de la dinámica estacional de la micorrización bajo regímenes contrastante de pastoreo. Agroagencia Uruguay, 15 (2): 1-10

Biovirtual. (2010). Clasificación Taxonómica del pasto (Panicum Maximun Jaqc) Instituto de Ciencia Naturales. Bogotá. Colombia.
Ecuared. (2012). Clasificación Botánica del Pasto Brachiaria Brizantha (Hochst. Ex A. Rich) Stapf y Digitaria Decumbens Stent. Recuperado en: www.ecuared.cu/index.php/Brachiaria_brizantha

Enciclopedia Práctica de la Agricultura y la Ganadería. (1999). Cultivos herbáceos extensivo. Barcelona-España. 483 p.

Haro, R. (2003). I Informe sobre Recursos Zoogenéticos Ecuador. (en línea): ftp://ftp.fao. org/docrep/fao/011/a1250f/annexes/CountryReports/Ecuador.pdf.

García, J. (2017). Evaluación del efecto agronómico de hongos micorrícicos arbusculares en el desarrollo vegetativo del cultivo de banano (Musa acuminata AAA) a nivel de campo, en la zona de Babahoyo, provincia de Los Ríos, tesis para optar por el grado de ingeniero Agropecuario, Universidad Guayaquil, Ecuador, 2017.

INEC (Instituto Nacional de Estadística y Censos (2013), (en línea): www.inec.gob.ec/

MAGAP (Ministerio de Agricultura Ganadería Acuacultura y Pesca. (2012). Importancia de la Ganadería, (en línea): http://www.magap.gob. ec/

Maldonado, K; Reyes, M. (2018). Evaluación de cuatro cepas de micorriza vesículo arbuscular en los cultivos de tomate y pasto Marandú en macro túnel, tesis para optar por el grado de licenciado en agronomía, Escuela Zamorano, Honduras, P20

Mundo-Pecuario. (2012). Descripción del Pasto Panicum Maximun Jaqc. Recuperado en: http://mundo-pecuario.comtema191/gramineas/pasto_guinea-1057.html

Noda, Y. (2009). Las Micorrizas: Una alternativa de fertilización ecológica en los pastos. Estación Experimental de Pastos y Forrajes "Indio Hatuey". Central España Republicana, CP 44280, Matanzas, Cuba, P10. 
Paillacho, F. (2010). Evaluación de la efectividad de las micorrizas arbusculares nativas sobre el desarrollo y estado nutritivo del palmito (Bactris gasipaes HBK) en la etapa de vivero, en Santo Domingo de los Tsáchilas - Informe del proyecto de investigación presentado como requisito parcial para optar por el título de Ingeniero Agropecuario. Escuela Superior Politécnica del Ejercito, Santo Domingo de los Tsáchilas,.

Prieto, O; Belezaca, C; Mora, W; Vallejo, E; Gutiérrez V; Pinargote E. (2011). Inoculación de Brachiaria decumbens con hongos formadores de micorriza arbuscular nativos del Trópico húmedo ecuatoriano. Ciencia y Tecnología 4(2): 9-18.

Reinoso, R. (2014). Efecto de la sustitución parcial de fertilizante químico por Zeolita y Micorriza en la producción de Brócoli. (Brassica oleracea L Vr. Botrytis), [Tesis para optar por el título de ingeniero agrónomo], Escuela Superior Politécnica del ejército Cotopaxi Ecuador.

Reyes, I. (2011). La micorriza arbuscular (MA) centro de la rizosfera: Comunidad microbiológica dinámica del suelo Depto. de Biología, División de CBS. UAM-Iztapalapa. (en línea): http://www.izt.uam.mx/newpage/contactos/ revista/81/pdfs/micorriza.pdf

Tropicalforanges (2011) Descripción Botánica del Pasto Digitaria Decumbens Stent y Panicum Maximun: (en línea): htpp://www. tropicalforages.info/Multiproposito/Key/ Multiproposito/Media/Htlk/Brachiaria\%20decumbens\%20Stapf.htm

Simbiotica (2008) Redes Simbiótica de Biología y conservación. Recuperado de: http:// www.simbiotica.org/fungi.htm

Yao, Q; Zhu, H,H; Hu, Y.L \& Li, L.Q. (2008). Differential influence of native and introduced arbuscular mycorrhizal fungi on growth of dominant and subordinate plants. Plant Ecology. 196:261

\section{CORRESPONDENCIA:}

Dr. Henrry Othón Intriago Mendoza

henry.intriago@uleam.edu.ec 